\title{
Elastic criterion for dislocation nucleation
}

\author{
$\mathrm{Ju} \mathrm{Li}^{\mathrm{a}, \mathrm{c}}$, Ting Zhu ${ }^{\mathrm{a}}$, Sidney Yip ${ }^{\mathrm{a}, \mathrm{b}, *}$, Krystyn J. Van Vliet ${ }^{\mathrm{b}}$, Subra Suresh ${ }^{\mathrm{b}}$ \\ a Department of Nuclear Engineering, Massachusetts Institute of Technology, 77 Massachusetts Avenue, \\ Cambridge, MA 02139, USA \\ ${ }^{\mathrm{b}}$ Department of Materials Science and Engineering, Massachusetts Institute of Technology, \\ 77 Massachusetts Avenue, Cambridge, MA 02139, USA \\ ${ }^{\mathrm{c}}$ Department of Materials Science and Engineering, Ohio State University, Columbus, OH 43210, USA
}

\begin{abstract}
The notion of theoretical strength, the critical stress at which a perfect crystal under uniform loading becomes structurally unstable, is extended to non-uniform loading. A position-dependent defect nucleation criterion has been derived and applied in molecular dynamics (MD) and finite-element simulations of dislocation emission in single-crystal nanoindentation. The resulting measure has the physical meaning of a local stiffness; it provides a rigorous basis for modeling the incipient plasticity in a thin-film material. Furthermore, a close connection has been shown to exist between the initial unstable elastic wave and the final atomistic defect.
\end{abstract}

(c) 2003 Elsevier B.V. All rights reserved.

Keywords: Nanoindentation; Ideal strength; Long wave; Defect nucleation

\section{Introduction}

In computational materials research multiscale modeling has come to symbolize the linking of simulation models describing phenomena on length and time scales from microscopic to macroscopic. In this paradigm electronic structure would be treated by quantum-mechanical calculations, atomistic processes by molecular dynamics (MD) or Monte Carlo simulations, microstructure evolution by methods such as dislocation dynamics or kinetic Monte Carlo, and continuum behavior by field equations well known in fluid and solid mechanics. The appeal of such an approach stems from the belief that by combining the different methods, one can attack problems of longstanding interest in a much more comprehensive manner than where the methods are used individually. Multiscale modeling is most useful when it allows us to gain physical insights not accessible by experiment or theory-on-paper. The fact that in a simulation one has direct control over the initial and boundary conditions and access to complete information on the electronic and molecular levels makes it possible to unravel the microscopic mechanisms underlying a partic-

\footnotetext{
* Corresponding author. Tel.: +1-617-253-3809; fax: +1-617-258-8863.

E-mail address: syip@mit.edu (S. Yip).
}

ular phenomenon. A coherent combination of experiments with modeling is also the most promising way to achieve systematic structure-property correlations.

Nanoscale indentation experiments are currently used to probe the mechanical response of crystals subjected to large local contact forces. Such contacts can induce elastic instabilities at weak spots inside the sample leading to the homogeneous nucleation of one or more dislocations. The major obstacle to a quantitative interpretation of these experiments is the lack of a theoretical description relating elastic instability to dislocation nucleation. During the past year we have derived a defect nucleation criterion and demonstrated its validity through molecular dynamics and finite-element simulations. As a result, a self-consistent explanation of the discontinuous elastic-plastic response observed in nanoindentation measurements is now available, as well as a guide to mechanical behavior studies seeking to quantify and predict the strength of materials at small length-scales.

\section{Theoretical strength: the elastic limit}

The theoretical basis for describing the strength of a crystal lattice lies in the formulation of stability conditions which specify the critical level of external stress that the system can withstand. Born has shown that by expanding the internal energy of a crystal in a power series in the strain and 
requiring positivity of the energy, one obtains a set of conditions on the elastic constants of the crystal that must be satisfied if the lattice is to maintain structural stability $[1,2]$. This then leads to a determination of ideal strength of perfect crystals as an instability phenomenon, a concept later examined by Hill [3] and Hill and Milstein [4], as well as used in various applications [5]. We have shown that Born's results are valid only when the solid is under no load, by invoking the formulation of a Gibbs integral [6]. Further discussions were given by Zhou and Joos [7] and by Morris and Krenn [8], the latter emphasizing the thermodynamic basis of the concept of theoretical strength by showing the generalized stability criteria [6] are consistent with the internal conditions for elastic stability formulated by Gibbs in 1876. A consequence of these investigations is that theoretical strength should be considered a property which can be affected by the symmetry and magnitude of the applied load, rather than an intrinsic property of the material. In this respect the study of theoretical limits to material strength using atomistic models, including first-principles calculations [9], has the potential to yield new insights into mechanisms of structural instability.

Under a finite, constant, external stress in the applied force-per-area sense the elastic stability criterion takes on the form $\operatorname{det}\left[\boldsymbol{B}+\boldsymbol{B}^{\mathrm{T}}\right]>0$, where $\boldsymbol{B}$ is the elastic stiffness tensor:

$B_{i j k l}=C_{i j k l}+\frac{1}{2}\left(\delta_{i k} \sigma_{j l}+\delta_{j k} \sigma_{i l}+\delta_{i l} \sigma_{j k}+\delta_{j l} \sigma_{i k}-2 \delta_{k l} \sigma_{i j}\right)$

with $C_{i j k l}$ being the isothermal elastic constant to be evaluated in the current state, and $\sigma_{i j}$ being the external stress. In the case of cubic crystals under a hydrostatic stress, the criteria are particularly simple:

$$
\begin{aligned}
& C_{11}+2 C_{12}+P>0, \quad C_{11}-C_{12}-2 P>0, \\
& C_{44}-P>0
\end{aligned}
$$

with the elastic constants now expressed in Voigt notation, and $P>0(<0)$ denotes compression (tension). We have found these criteria to be useful in molecular dynamics studies of stress-induced polymorphic transition in $\mathrm{Si}$ [10], cleavage fracture in $\mathrm{SiC}$ [11], and pressure-induced amorphization in $\mathrm{SiC}$ [12] and $\mathrm{SiO}_{2}$ [13].

Eq. (1) is the Jacobian matrix of the Gibbs free energy (or path-integral) with respect to further deformation, and is only applicable when the entire coarsely homogenous block of material is in direct contact with externally applied forcing, such as when the block is deep in ocean so the applied pressure on the surface can be taken to be a literal constant in the force-per-area sense. This way, if the block undergoes a virtual deformation, it would be able to elicit work from the external environment, and the Gibbs free energy will be the governing thermodynamic potential. However, we must caution that this setup is rather singular and can be regarded as a degenerate case of the more general non-uniform loading condition, and therefore deserves special care. There are subtleties such as: (a) one would arrive at a different criterion from Eq. (1) if the external forcing is dead load instead of constant stress in the force-per-area sense; and (b) $B$ is asymmetric with respect to $i j \leftrightarrow k l$ exchange under non-hydrostatic loading, reflecting the path-dependence of the Gibbs integral, which all stems from the above degeneracy.

The foregoing elastic stability criterion applies to a perfect crystal block under homogeneous deformation. For a perfect crystal under inhomogeneous deformation, one can expect one or more defects to be nucleated at internal sites-defined to be the weak spots-when the loading reaches a critical level. This problem was first discussed by Hill [14] in a study of "acceleration waves", a dynamical, continuum analysis where the acceleration across a surface becomes discontinuous. Rice treated essentially the same instability phenomenon [15] in the context of shear localization, arriving at a formal criterion with a tensor $\boldsymbol{L}$ playing a similar role as the stiffness tensor $\boldsymbol{B}$ in Eq. (2).

We have derived a defect nucleation criterion which is in effect both an extension and a practical implementation of the formal results of Hill and Rice. We consider a representative volume element (RVE) subjected to homogeneous deformation at finite strain to a current configuration $\boldsymbol{x}$. Expanding the free energy $F$ to second-order in incremental displacement $u(\boldsymbol{x})$, we obtain

$$
\Delta F=\frac{1}{2} \int_{V(\boldsymbol{x})} D_{i j k l} u_{i, j}(\boldsymbol{x}) u_{k, l}(\boldsymbol{x}) \mathrm{d} V
$$

where $D_{i j k l}=C_{i j k l}+\tau_{j l} \delta_{i k}, \tau_{j l}$ being the internal (Cauchy) stress, and $u_{i, j} \equiv \partial u_{i}(\boldsymbol{x}) / \partial x_{j}$. Representing the displacement by a plane wave perturbation, $u_{i}(\boldsymbol{x})=w_{i} \mathrm{e}^{\mathrm{i} \boldsymbol{k} \cdot \boldsymbol{x}}$, leads to the stability condition for the RVE:

$\Lambda(\boldsymbol{w}, \boldsymbol{k}) \equiv\left(C_{i j k l} w_{i} w_{k}+\tau_{j l}\right) k_{j} k_{l}>0$

The sign of $\Lambda$ reflects the concavity of $F$. If there exists a pair of $\boldsymbol{w}, \boldsymbol{k}$ such that $\Lambda$ is negative, then homogeneity of this RVE cannot be maintained and defect singularities will form internally. In other words, the elastic stability of the RVE can be determined by minimizing $\Lambda$ with respect to the polarization vector $\boldsymbol{w}$ and the wave vector $\boldsymbol{k}$; the minimum value of $\Lambda, \Lambda_{\min }$, therefore provides a measure of the micro-stiffness locally, with instability predicted at the position where $\Lambda_{\min }$ vanishes. Notice that Eq. (4) is an energy-based criterion; its minimization at a material point is dependent on the local stress environment. Also, the Helmholtz instead of the Gibbs free energy is expanded because we assume either (a) the plane wave perturbations reside in a periodic boundary condition box (the Born-von Karman condition) so external work cancels out; or (b) the long elastic waves constitute a wave packet that is localized inside the weak spot which is not in contact with the external forcing, so the perturbation would not elicit external work. Actual nanoindentation experiments using smooth spherical indenters correspond to the second scenario, because we know from Hertzian analysis that the maximum 
shear stress does not occur at the contact surface but is at a finite depth beneath the indenter (similar to a lens' optical focus), so the weak spot develops within the material that is not in contact with the indenter, so naturally the instability mode would localize inside the weak spot, and not venture outside to domains with positive micro-stiffness $\Lambda_{\min }$, because it does not pay free-energy-wise anyway. With vanishing displacement field everywhere outside, the mode would not elicit work from contact forces, and therefore when analyzing the free energy balance, we need not take external work into account.

One may regard the elastic stability criterion (Eq. (4)) as the condition for vibrational stability of a perfect crystal lattice in the long wavelength limit. The vanishing of $\Lambda_{\text {min }}$ then corresponds to the phenomenon of soft phonon modes in lattice dynamics. Indeed one finds under sufficient deformation such soft modes do occur in a homogeneously strained lattice. Fig. 1 depicts the correspondence between the softening of phonon frequencies in fcc $\mathrm{Al}$ and the stress-strain response of the single-crystal system at low temperature, both simulated using the Ercolessi-Adams potential [26] and undergoing hydrostatic strain until beyond the limit of ideal strength. Dispersion curves, obtained by diagonalizing the dynamical matrices in $\boldsymbol{k}$-space, are shown along with a stress-strain response determined by molecular dynamics simulation using a supercell containing 4000 atoms at $T=10 \mathrm{~K}$ under $\mathrm{PBC}$, corresponding to the first scenario mentioned in the last paragraph. Results for the undeformed lattice are given in the upper panels, along with experimentally measured phonon dispersion data obtained by neutron scattering [27]. In the lower panels the system is at the state of critical strain as indicated on the stress-strain curve. It can be seen from in the lower left panel that certain phonon modes near the $\Gamma$-point have just become unstable.

The soft phonon analysis, when all modes including the zone-boundary modes are considered [28], guarantees the structural stability of a perfect crystal as $T \rightarrow 0$ because the phonons constitute a complete basis for all atomic motion in the system. But at finite temperature we no longer have
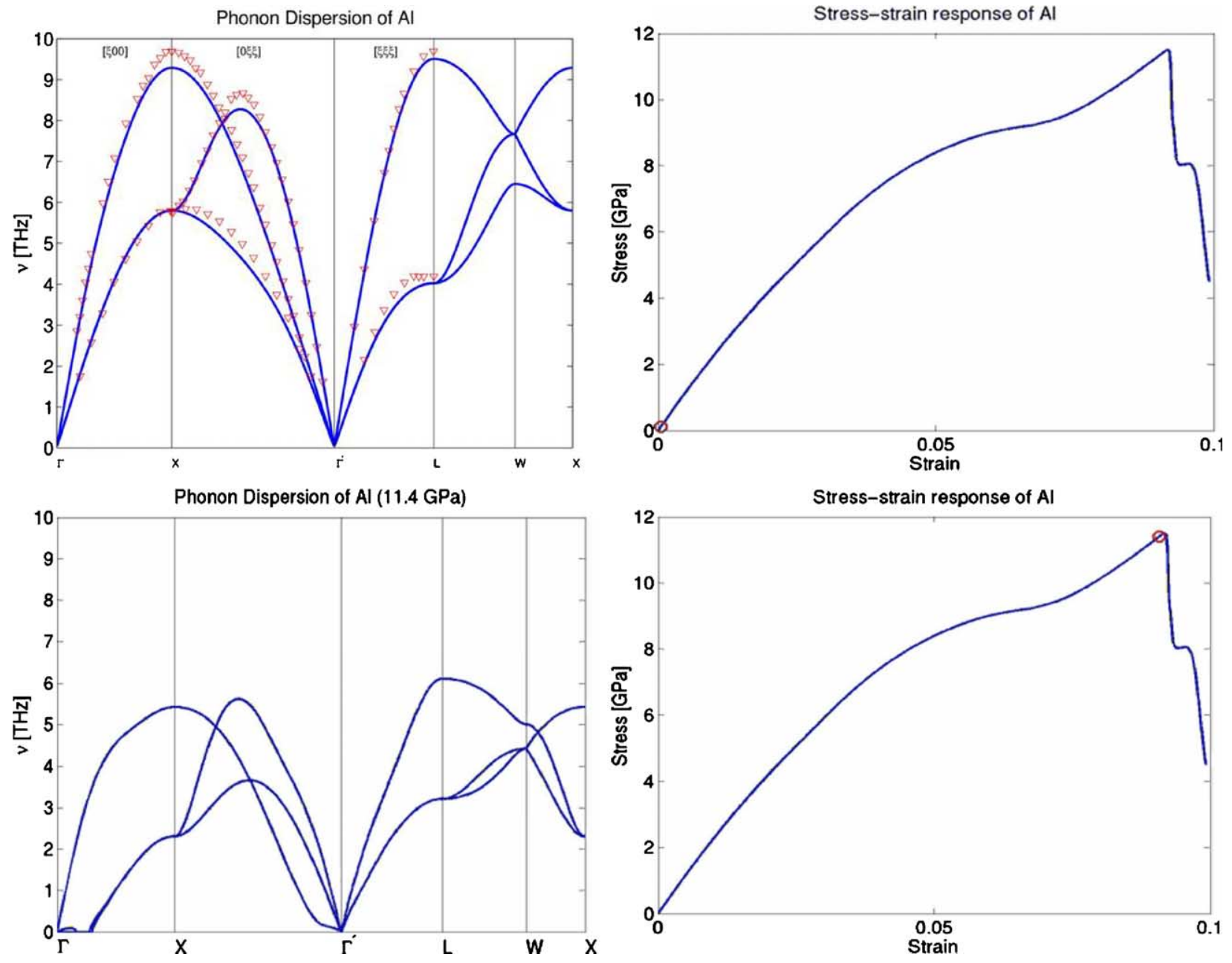

Fig. 1. Phonon dispersion curves and stress-strain response of a single-crystal fcc Al under hydrostatic tension. Undeformed results (upper panels) and results in the vicinity of critical strain (lower panels), both denoted by the circle on the stress-strain curve. Experimentally measured phonon dispersion data by neutron scattering are shown as triangles. 
the above exact criterion, because energies of the modes are no longer decoupled, in which case the quasi-harmonic approximation is a useful but no longer exact tool. On the other hand, although Eq. (4) is but a special case of the full soft phonon analysis at $T=0$, it remains a rigorous criterion at finite temperature because of its thermoelastic origin. We believe that Eq. (4) covers most of the instabilities driven by affine strain in monatomic crystals, whereas full phonon analysis is usually necessary for more complicated crystals such as $\beta-\mathrm{SiC}[28]$.

\section{Application of $\boldsymbol{\Lambda}$-criterion to nanoindentation}

The implementation of the defect nucleation criterion is nontrivial. There are conceptual and computational issues to be resolved in demonstrating the practical utility of our formulation. The conceptual challenge is the lack of accurate constitutive models to estimate $\tau_{j l}$ and $C_{i j k l}$ at strains great enough to trigger local structural instability. (How best to represent the strain energy in the six-dimensional space of general strain using ab initio calculations? By brute force, it would require $\sim 10^{6}$ spline data to be evaluated even with merely 10 mesh points in one dimension.) The computational challenges also include how to calculate $\Lambda_{\min }$ reliably. While minimization in the four-dimensional space of $\boldsymbol{k}$ and $\boldsymbol{w}$, with each being a unit vector, is not an especially difficult task, doing it at every material point in the system requires an exceptionally reliable and efficient algorithm. For example, our determination of $\Lambda_{\text {min }}$ in the molecular dynamics simulation of nanoindentation involves the calculation of this quantity for each atom at every timestep, with the simulation consisting of up to 2 million atoms and runs as long as $10^{5}$ steps.

In applying the criterion to nanoindentation of thin films, our intention is to probe the elastic limit and incipient plasticity at critical loading [16,17]. Two modes of implementation are developed, one is direct molecular dynamics simulation and the other involves a new method which incorporates interatomic potential calculations in a finite-element framework. In the latter the problem of obtaining a suitable constitutive model is solved by invoking the Cauchy-Born condition $[18,19]$ which then allows atomistic simulations on-the-fly to return the local stress and elastic constants [20,21] for a given nodal strain. Further details are given below. For pair and embedded-atom type interatomic potentials, the partitioning of lattice sums into contributions from individual atoms is straightforward, whereas for more complicated potential forms special arrangements will be needed to preserve symmetry and sum-rule requirements [22]. The partitioning is justified as long as the atom in question is a few lattice spacing away from any topological defects, even if the atom is situated in a field of large affine strain.

To overcome the computational challenge we note that $\Lambda(\boldsymbol{k}, \boldsymbol{w})$ is a bi-quadratic function in $\boldsymbol{k}$ and $\boldsymbol{w}$, and can be alternately written as

$$
\Lambda(\boldsymbol{w}, \boldsymbol{k})=\left(C_{i j k l} k_{j} k_{l}\right) w_{i} w_{k}+\tau_{j l} k_{j} k_{l}
$$

where the order of $\boldsymbol{k}, \boldsymbol{w}$ summation is exchanged from Eq. (4). If we assume $\boldsymbol{w}$ were a constant in Eq. (4) and minimize with respect to $\boldsymbol{k}$ only, then solving the simple quadratic minimization involves diagonalizing the $3 \times 3$ symmetric matrix $[\boldsymbol{A}]_{j l} \equiv C_{i j k l} w_{i} w_{k}+\tau_{j l}$ and choosing the

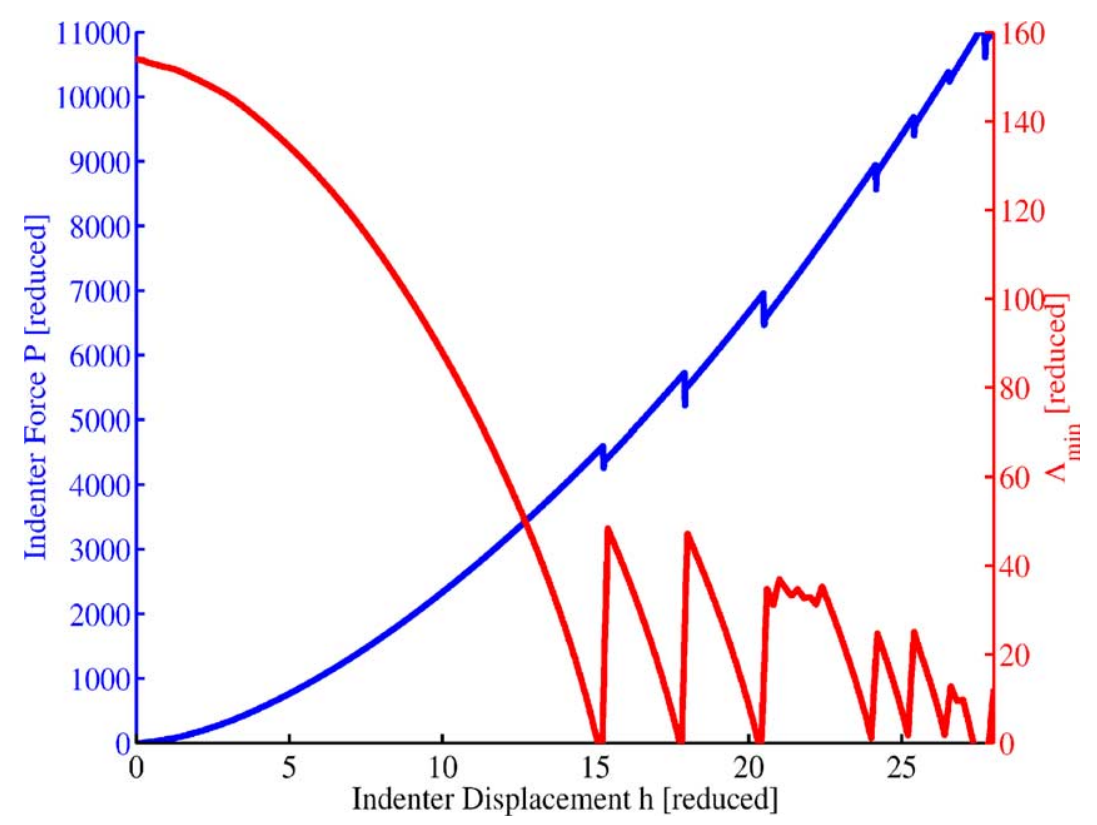

Fig. 2. Molecular dynamics results for the load-displacement curve (displacement controlled) in nanoindentation on bubble raft showing characteristic load drops when a dislocation is nucleated [16], and variation of $\Lambda_{\min }$ with indenter depth. 


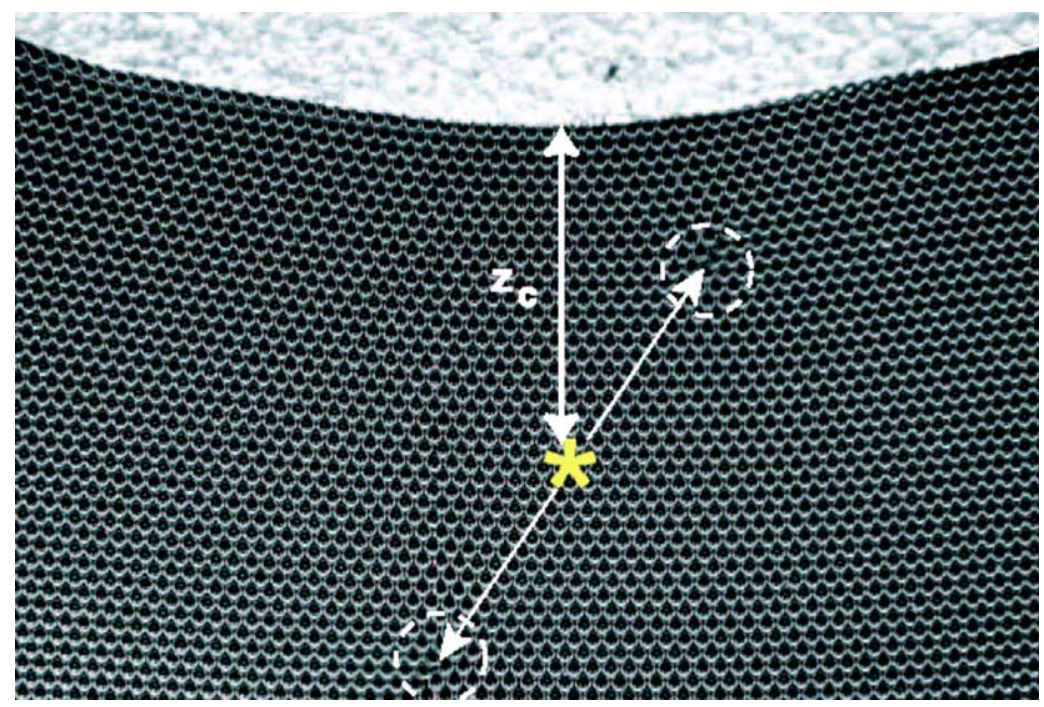

Fig. 3. Indentation of Bragg-Nye soap bubble raft causing the nucleation (position denoted by $*$ ) of an edge dislocation dipole which dissociates in the manner indicated by the arrows [17]. Nucleation occurs at a distance $z_{0}$ which scales at 0.78 of the indenter radius.

softest eigenvector to be $\boldsymbol{k}$. Alternately, assuming $\boldsymbol{k}$ to be a constant in Eq. (5) we can minimize with respect to $\boldsymbol{w}$, and the solution is again straightforward, diagonalizing $[\boldsymbol{B}]_{i k} \equiv C_{i j k l} k_{j} k_{l}$ and choosing the softest eigenvector to be $\boldsymbol{w}$. Based on these observations an iterative algorithm to minimize $\Lambda(\boldsymbol{w}, \boldsymbol{k})$ consists of repeating the sequence-choose arbitrary $\boldsymbol{w}$, minimize Eq. (4) for a fixed $\boldsymbol{w}$ to obtain an initial $\boldsymbol{k}$, then minimize Eq. (5) keeping $\boldsymbol{k}$ fixed to obtain a new vector $\boldsymbol{w}$-until convergence. Since only diagonalizations of $3 \times 3$ matrices are involved, the algorithm can be made quite efficient by using the analytical solution of a cubic algebraic equation [23].

Fig. 2 shows the load-displacement curve of a displacement-controlled nanoindentation of a two-dimensional system in the form of Bragg-Nye bubble rafts $[16,17]$. The MD results, obtained by molecular dynamics simulation using an appropriate inter-bubble potential, show a series of load drops, each signifying the nucleation of a dislocation. An event of this kind is seen in Fig. 3 which is an actual image from a bubble-raft experiment [17], where a dislocation dipole is nucleated subsurface at a depth predicted by continuum (Hertzian) contact mechanics to scale with the radius of the indenter. Also shown in Fig. 2 are the results for $\Lambda_{\min }$, evaluated for the bubble-raft simulation system in the manner described above. One sees a close correspondence between the sequence of load drops observed in the MD simulation and the sequence of vanishing of $\Lambda_{\min }$ over a range of indenter displacements. Moreover, we find that the $\boldsymbol{w}$ and $\boldsymbol{k}$ vectors which minimize $\Lambda(\boldsymbol{w}, \boldsymbol{k})$ agree well with the slip direction and slip plane normal, respectively, observed in the MD simulation. We regard this agreement between direct simulation and nucleation-criterion analysis to be a quantitative validation of our formulation and implementation. Before leaving Fig. 2 one should also note that as the indentation loading approaches the first load drop, the system response is purely elastic, and the corresponding values of $\Lambda_{\text {min }}$ monotonically decreases toward zero, thus confirming the meaning of local micro-stiffness that we have ascribed to $\Lambda_{\min }$.

Because the instabilities predicted by the $\Lambda$-criterion are elastic in nature, and since all atomic-level information is incorporated through $C_{i j k l}$ and $\tau_{j l}$, our defect nucleation criterion also can be implemented in the framework of finite-element method. Specifically, we have applied the code ABAQUS in the finite-strain mode with a constitutive relation provided by making use of the Cauchy-Born rule $[18,19]$. In this description each material point (node) is represented locally by an infinite lattice subjected to a homogeneous deformation, prescribed by the current state of the finite-element calculation. The nodal stress is then

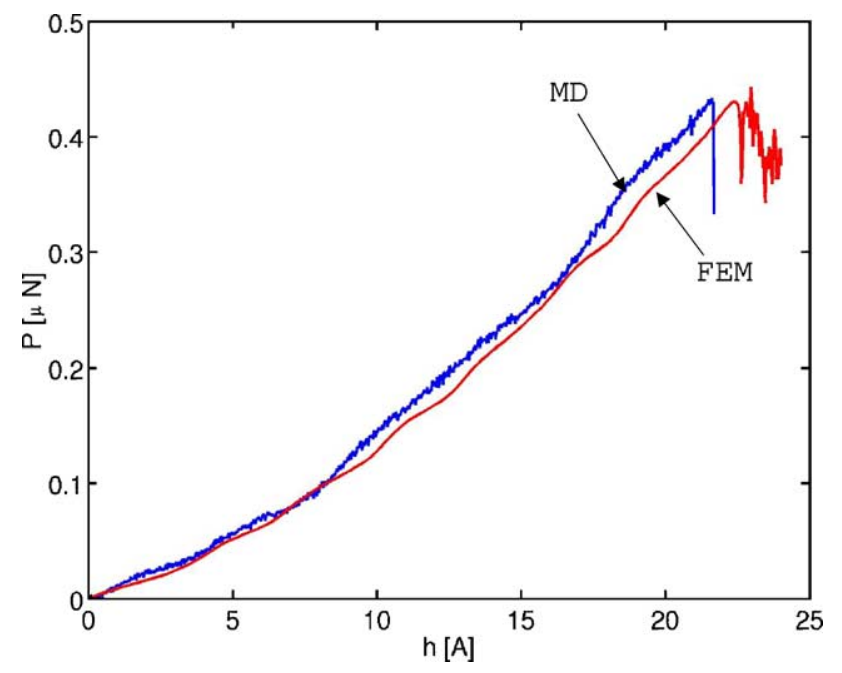

Fig. 4. Comparison of load-displacement responses in two-dimensional nanoindentation simulated by MD and by IPFEM using the same interatomic potential as the MD [16]. 
calculated from the deformed lattice using an appropriate interatomic potential function. This procedure can be applied at every node in the finite-element model, in the same way that the defect nucleation criterion is evaluated at every atom in the system in the molecular dynamics implementation. A direct test of this scheme, which we have called IPFEM, against pure MD simulation in the case of two-dimensional nanoindentation, is shown in Fig. 4.

\section{Discussion}

We developed the $\boldsymbol{B}$-criterion for constant stress loading under uniformly distributed strain, and there are other criteria for dead load conditions, etc. which are all based on taking the second-order derivatives of the Gibbs free energy. On the other hand, the $\Lambda$-criterion is based on taking the second-order derivatives of the Helmholtz free energy, which can be applied to cases of non-uniform loading in which a weak spot develops inside the material that is not in direct contact with the external forces and so we do not need to consider external work contribution. Depending on which description is really applicable, we arrive at two different criteria for elastic instabilities. This loading condition dependence differs from a well-known property of the fracture instability criterion in terms of the fracture toughness, where it has been shown that whether the external loading condition is constant force or displacement does not matter. The reason for this discrepancy is that elastic instabilities are second-order instabilities, where the perturbed variable is the affine strain and the energy of concern scales as strain squared, so we need to do variational calculus accurate to the second-order, whereas in the formulation of the fracture criterion, the variable is crack extension, and the energy of concern (surface energy minus strain energy release rate) scales linearly. As a general observation, all first-order instabilities are insensitive to the exact specification of the loading condition, but second-order instabilities may be sensitive.

The soft phonon modes we discussed have eigenvectors which allow one to determine the character of the elastic instability. This analysis extends naturally to translational symmetry-broken systems where the wavevector $\boldsymbol{k}$ is no longer a good label of all the normal modes in the dynamical system; this has been formulated in terms of real-space Green's function and applied to analyze the onset of fracture in a strained lattice $[24,25]$. In this respect, it would be interesting to generalize the $\Lambda$-criterion to the problem of crack-tip extension.

We are continuing to seek a more fundamental understanding of how a strained lattice, initially homogeneous, is driven to nucleate a defect. An interesting question is: what is the process by which a smooth wave in the continuum metamorphoses into an atomistic defect which is a singularity if seen at the continuum level? We believe the process is characterized as a sequence of four stages. (1) An unstable elastic wave grows exponentially with time as indicated by $\Lambda_{\min }<0$, without changing its shape. (2) As the wave amplitude increases to a significant level, growth becomes nonlinear and the wave profile steepens progressively. (3) Steepening becomes singular on the continuum level and the wave front is resolvable only on the atomic scale. (4) Atomistically sharp wave front is stabilized as it is trapped in a local minimum in a low-dimensional atomic energy landscape such as the $\gamma$-surface. There are also intriguing correlations between the affine strain and the highly localized strain energy landscapes [29].

\section{Acknowledgements}

This work was sponsored by ONR Grant N00014-01-0808. J.L., T.Z. and S.Y. acknowledge support by AFOSR Grant F49620-00-10082, Honda R\&D, NSF (DMR-9980015 and ITR). K.J.V.V. acknowledges support of the National Defense Science and Engineering Graduate Fellowship program.

\section{References}

[1] M. Born, Proc. Cambridge Philos. Soc. 36 (1940) 160.

[2] M. Born, K. Huang, Dynamical Theory of Crystal Lattices, Clarendon Press, Oxford, 1956.

[3] R. Hill, Math. Proc. Cambridge Philos. Soc. 77 (1975) 225.

[4] R. Hill, F. Milstein, Phys. Rev. B 15 (1977) 3087.

[5] A. Kelly, N.H. Macmillan, Strong Solids, third ed., Clarendon Press, Oxford, 1986.

[6] J. Wang, J. Li, S. Yip, S. Phillpot, D. Wolf, Phys. Rev. B 52 (1995) 12627.

[7] Z. Zhou, B. Joos, Phys. Rev. B 54 (1996) 3841.

[8] J.W. Morris, C.R. Krenn, Philos. Mag. A 80 (2000) 2827.

[9] J.W. Morris, C.R. Krenn, D. Roundy, M.L. Cohen, in: P.E. Turchi, A. Gonis (Eds.), Phase Transformations and Evolution in Materials, TMS, Warrendale, 2000, p. 187.

[10] K. Mizushima, S. Yip, E. Kaxiras, Phys. Rev. B 50 (1994) 14952.

[11] M. Tang, S. Yip, J. Appl. Phys. 76 (1994) 2719.

[12] M. Tang, S. Yip, Phys. Rev. Lett. 75 (1995) 2738.

[13] S. Subraramian, D. Liao, S. Yip, to be published.

[14] R. Hill, J. Mech. Phys. Solids 10 (1962) 1.

[15] J.R. Rice, in: W.T. Koiter (Ed.), Theoretical and Applied Mechanics, North-Holland, Amsterdam, 1976, p. 207.

[16] J. Li, K.J. Van Vliet, T. Zhu, S. Suresh, S. Yip, Nature 418 (2002) 307

[17] K.J. Van Vliet, J. Li, T. Zhu, S. Yip, S. Suresh, Phys. Rev. B 67 (2003) 104105

[18] F. Milstein, J. Mater. Sci. 15 (1980) 1071.

[19] M. Ortiz, R. Phillips, Adv. Appl. Mech. 36 (1999) 1.

[20] T. Egami, K. Maeda, V. Vitek, Philos. Mag. A 41 (1980) 883.

[21] J.R. Ray, Comp. Phys. Rep. 8 (1988) 109.

[22] J. Li, L.J. Porter, S. Yip, J. Nucl. Mater. 255 (1998) 139.

[23] G. Cardano, Ars Magna. Milan (1545).

[24] C.S. Jayanthi, M. Tang, S.Y. Wu, J.A. Cocks, S. Yip, Phys. Rev. Lett. 79 (1997) 4601.

[25] J. Li, S. Yip, Phys. Rev. B 56 (1997) 3524.

[26] F. Ercolessi, J.B. Adams, Europhys. Lett. 26 (1994) 583.

[27] R. Stedman, G. Nilsson, Phys. Rev. 145 (1966) 492.

[28] A. Romano, J. Li, S. Yip, Physica A 304 (2002) 11.

[29] S. Ogata, J. Li, S. Yip, Science 298 (2002) 807. 